\title{
The motion of a rigid body and a viscous fuid in a bounded domain in presence of collisions
}

\begin{abstract}
We consider the motion of a rigid body in a bounded domain filled by viscous incompressible fluid. The fluid is described by the Navier-Stokes equations. We assume the Navier condition on the boundary of the body and the Dirichlet condition on the boundary of the domain. We give the global-in-time solvability result of the weak solution. The result allows a possibility of collisions of the body with the boundary of the domain.
\end{abstract}

Keywords: navier-stokes equations, navier boundary condition, solidification
Received: July 13, 2017| Published: January 03, 2018

\section{Presentation of the problem}

We investigate the motion of a rigid body inside a viscous incompressible fluid. Let $\Omega$ be a bounded domain of $\mathbb{R}^{N}$ for $N=2$ or 3. At the initial moment $t=0$ the body and the fluid occupy an open connected set $S_{0} \subset \Omega$ and the set $F_{0}=\Omega \backslash S_{0}$, respectively. The motion of any point $\mathbf{y}=\left(y_{1}, . ., y_{N}\right)^{T} \in S_{0}$ is described by an isometry, preserving orientation.

$$
\mathbf{A}(t, \mathbf{y})=\mathbf{q}(t)+\mathbb{Q}(t)(\mathbf{y}-\mathbf{q}(0)), \quad t \in 0, T],
$$

where $\mathbf{q}=\mathbf{q}(t)$ is the body mass center and $\mathrm{Q}(\mathrm{t})$ is the rotation matrix, such that $\mathbb{Q}(t) \mathbb{Q}(t)^{T}=\mathbb{I}, \mathbb{Q}(0)=\mathbb{I}$ with $\mathbb{I}$ being the identity matrix. Hence the body and the fluid occupy the sets $S(t)=A\left(t, S_{0}\right)$ and $F(t)=\Omega \backslash S(t)$ at any time $t$. The velocity of the body is related with the isometry $\mathbf{A}$ by

$$
\mathbf{u}=\mathbf{q}^{\prime}(t)+\mathbb{P}(t)(\mathbf{x}-\mathbf{q}(t)) \quad \text { for } \mathbf{x} \in S(t),
$$

where the matrix $\mathbb{P}(t)$ fulfills $\frac{d \mathbb{Q}}{\mathbb{Q}} \mathbb{Q}^{T}=\mathbb{P}$, such that there exists a vector $\omega=\omega(t) \in \mathbb{R}^{N}$, satisfying $d \mathbb{P}(t) \mathbf{x}=\omega(t) \times \mathbf{x}, \quad \forall \mathbf{x} \in \mathbb{R}^{N}$.

Let $\rho$ be the density of the body $\mathrm{S}(\mathrm{t})$ and of the fluid $F(t)$ at a time $t \in 0, T]$. Then the mass of the body $S(t)$ is equal to $m=\int_{S(t)} \rho d \mathbf{x}$ . The stress and the deformation-rate tensors of the fluid are given by

$$
P=-p I+2 \mu_{f} \mathbb{D} \mathbf{u} \text { and } \mathbb{D} \mathbf{u}=\frac{1}{2}\left\{\nabla \mathbf{u}+(\nabla \mathbf{u})^{T}\right\},
$$

where $p$ is the fluid pressure and the viscosity $\mu_{f}$ of the fluid is a positive constant. The matrix of the inertia moments of the body is calculated as

$$
\mathbb{J}=\int_{S(t)} \rho\left(|\mathbf{x}-\mathbf{q}(t)|^{2} \mathbb{I}-(\mathbf{x}-\mathbf{q}(t)) \otimes(\mathbf{x}-\mathbf{q}(t))\right) d \mathbf{x} .
$$

Therefore the motion of the fluid and of the body is governed by the following system

$$
\begin{gathered}
\partial_{t} \rho+(\mathbf{u} \cdot \nabla) \rho=0, \quad \operatorname{div} \mathbf{u}=0, \quad \rho\left(\partial_{t} \mathbf{u}+(\mathbf{u} \cdot \nabla) \mathbf{u}\right)=\operatorname{div} P+\mathbf{g} \quad \text { for } \mathbf{x} \in F(t), \\
m \mathbf{q}^{\prime \prime}=\int_{\partial S(t)} P \mathrm{n} d \mathbf{x}+\int_{S(t)} \mathbf{g} d \mathbf{x}, \\
\frac{d(\mathbb{J} \omega)}{d t}=\int_{\partial S(t)}(\mathbf{x}-\mathbf{q}(t)) \times P \mathrm{n} d \mathbf{x}+\int_{S(t)}(\mathbf{x}-\mathbf{q}(t)) \times \mathbf{g} d \mathbf{x} \quad \text { for } \mathbf{x} \in S(t)
\end{gathered}
$$

with the initial conditions

$$
S=S_{0}, \quad \rho=\rho_{0}, \quad \mathbf{u}=\mathbf{u}_{0} \quad \text { at } t=0 .
$$

In system (3) $\mathrm{n}$ is the unit outward normal to $\partial S(t)$ and $\mathbf{g}$ is an external force.

The global existence of weak solutions of rigid bodies moving in a fluid has been investigated by many mathematicians: Hoffmann ${ }^{1}$ Conca $\mathrm{C}$ et al. ${ }^{2}$ Feireisl E et al., ${ }^{3}$ Bost $\mathrm{C}$ et al., ${ }^{4}$ Desjardins $\mathrm{B}$ et al. ${ }^{5}$ Gunzburger MD et al., ${ }^{6}$ Takahashi T et al., ${ }^{7}$ Judakov NV ${ }^{8}$ and etc.. All of these authors have considered the non-slip boundary condition on the boundaries of the body and the domain, although this boundary condition gives a paradoxical result of no collisions between the body and the boundary of the domain..$^{9-11}$ In the articles, ${ }^{11-13}$ the authors have studied the question of possible collisions with respect of the regularity of velocity and the regularity of boundaries. For instance, in Gérard Varet et al. ${ }^{12}$ have demonstrated that under $C^{1, \alpha}$-boundaries the collision is possible in a finite period of time if and only if $\alpha<1 / 2$. These mentioned results have demonstrated that a more accurate model is needed for the description of the motion of bodies in a viscous incompressible fluid.

Another possibility to include collisions is to consider the slippage on the boundaries. The slippage is prescribed by the Navier type of boundary condition. It means that there is only the continuity of velocity field in the normal component. A particular case, when the motion of a rigid body is prescribed, has been considered by Neustupa et. ${ }^{14}$ They have investigated a prescribed collision of a ball with a wall, when the slippage is allowed on the boundaries of the ball and of the wall. This pioneer result ${ }^{14}$ has shown that the slip boundary condition cleans the no-collision paradox. First result in the case of the motion of a single body, moved in the whole space $\mathbb{R}^{3}$, has been considered in G Planas ${ }^{15}$ Recently Gérard-Varet et al., ${ }^{16}$ have proved a local-in-time existence result: up to collisions. The free fall of a ball with assumptions on symmetry and touching point has been studied in D Gérard Varet ${ }^{17}$ In this article it has been shown that the ball touches the boundary of the wall in a finite period of time in the case of Navier boundary conditions on the boundaries of the ball and the wall.

In this article we close system (3) by adding Navier boundary condition 


$$
\mathbf{u}_{s} \cdot \mathrm{n}=\mathbf{u}_{f} \cdot \mathrm{n}, \quad\left(P \mathrm{n}+\gamma\left(\mathbf{u}_{f}-\mathbf{u}_{s}\right)\right) \cdot \mathrm{s}=0 \quad \text { on } \partial S(t),
$$

and Dirichlet boundary condition

$$
\mathbf{u}=0 \quad \text { on } \partial \Omega .
$$

Here $\mathbf{u}_{s}$ and $\mathbf{u}_{f}$ are the trace values of the velocity $\mathbf{u}$ on $\partial S(t)$ from the rigid side $S(t)$ and from the fluid side $F(t)$, respectively. The outer normal and arbitrary tangent vector to $\partial S(t)$ are denoted by $\mathrm{n}$ and $\mathrm{s}$. The constant $\gamma>0$ is a so-called friction coefficient of $\partial S_{0}$.

\section{Weak solution of system (I)-(6) and the main result}

To introduce the concept of weak solution for system (1)-(6), let us define some spaces of functions

$$
\begin{gathered}
V^{0,2}(\Omega)=\left\{\mathbf{v} \in L^{2}(\Omega): \operatorname{div} \mathbf{v}=0 \quad \text { in } \mathcal{D}^{\prime}(\Omega), \quad \mathbf{v} \cdot \mathrm{n}=0 \text { in } H^{-1 / 2}(\partial \Omega)\right\}, \\
B D_{0}(\Omega)=\left\{\mathbf{v} \in L^{1}(\Omega): \mathbb{D} \mathbf{v} \in \mathcal{M}(\Omega), \quad \mathbf{v}=0 \quad \text { on } \partial \Omega\right\},
\end{gathered}
$$

Where $n$ is the unit normal to the boundary $\partial \Omega$ of the domain $\Omega$ and $\mathcal{M}(\Omega)$ is the space of bounded Radon measures. Let $S$ be an open connected subset of $\Omega$. We consider the space

$K B(S)=\left\{\mathbf{v} \in B D_{0}(\Omega): \mathbb{D} \mathbf{v} \in L^{2}(\Omega \backslash \bar{S}), \quad \mathbb{D} \mathbf{v}=0 \quad\right.$ a.e.on $S, \quad \operatorname{div} \mathbf{v}=0 \quad$ in $\left.\mathcal{D}^{\prime}(\Omega)\right\}$

In what follows we admit that the boundary $\partial \Omega \in C^{0,1}$ of the domain $\Omega$ and the boundary $\partial S_{0} \in C^{2}$ of the rigid body $S_{0}$.

\section{Definition}

The triple $\{\mathbf{A}, \rho, \mathbf{u}\}$ is a weak solution of system (1)-(6), if the following three conditions are fulfilled:

1. The function $\mathbf{A}(t, \cdot): \mathbb{R}^{N} \rightarrow \mathbb{R}^{N}$ is an isometry (1), such that the functions $\mathbf{q}, \mathbb{Q}$ are absolutely continuous on $[0, T]$. The isometry A is compatible with the rigid body velocity (2) on $S(t)$ and defines a time dependent set $S(t)=\mathbf{A}\left(t, S_{0}\right)$;

2. The function $\rho \in L^{\infty}((0, T) \times \Omega)$ satisfies the integral equality

$$
\int_{0}^{T} \int_{\Omega} \rho\left(\xi_{t}+(\mathbf{u} \cdot \nabla) \xi\right) d t d \mathbf{x}=-\int_{\Omega} \rho_{0} \xi(0, \cdot) d \mathbf{x}, \quad \forall \xi \in C^{1}([0, T] \times \bar{\Omega}), \quad \xi(T, \cdot)=0 ;
$$

3. The function $\mathbf{u} \in L^{2}(0, T ; K B(S(t))) \cap L^{\infty}\left(0, T ; V^{0,2}(\Omega)\right)$ satisfies the integral equality

$$
\begin{aligned}
& \int_{0}^{T} \int_{\Omega \backslash \partial S(t)}\left\{\rho \mathbf{u y}_{t}+\rho(\mathbf{u} \otimes \mathbf{u}): \mathbb{D y}-2 \mu_{f} \mathbb{D} \mathbf{u}: \mathbb{D} \mathbf{y}+\mathbf{g y}\right\} d \mathbf{x} d t \\
& =-\int_{\Omega} \rho_{0} \mathbf{u}_{0} \mathrm{y}(0, \cdot) d \mathbf{x}+\int_{0}^{T}\left\{\int_{\partial S(t)} \gamma\left(\mathbf{u}_{s}-\mathbf{u}_{f}\right)\left(\mathrm{y}_{s}-\mathrm{y}_{f}\right) d \mathbf{x}\right\} d t
\end{aligned}
$$

for any $\mathrm{y} \in L^{2}(0, T ; K B(S(t)))$, such that $\mathrm{y}_{t} \in L^{2}\left(0, T ; L^{2}(\Omega \backslash \partial S(t))\right)$ and $\mathrm{y}(T, \cdot)=0$. Here we denote the trace values of $\mathbf{u}, \mathrm{y}$ on $\partial S(t)$ from the rigid side $S(t)$ and the fluid side $F(t)$ by $\mathbf{u}_{s}(t, \cdot), \mathrm{y}_{s}(t, \cdot)$ and $\mathbf{u}_{f}(t, \cdot), \mathrm{y}_{f}(t, \cdot)$, respectively.

Our main result is the following theorem shown in $\mathrm{NV} \mathrm{Chemetov}^{18}$ Theorem We assume that $\overline{S_{0}} \subset \Omega$. Let

$$
\rho_{0}(\mathbf{x})=\left\{\begin{array}{ll}
\rho_{s}(\mathbf{x}) \geqslant \text { const }>0, & \mathbf{x} \in S_{0}, \\
\rho_{f}=\text { const }>0, & \mathbf{x} \in F_{0},
\end{array} \quad \rho_{s} \in L^{\infty}\left(S_{0}\right),\right.
$$

$$
\mathbf{u}_{0} \in V^{0,2}(\Omega), \quad \mathbb{D} \mathbf{u}_{0}=0 \quad \text { in } \mathcal{D}^{\prime}\left(S_{0}\right), \quad \mathbf{g} \in L^{2}\left(0, T ;(K B(S(t)))^{*}\right) \text {. }
$$

Then for any given $T>0$ system (1)-(6) possesses a weak solution $\{\mathbf{A}, \rho, \mathbf{u}\}$, such that the isometry $\mathbf{A}(t, \cdot)$ is Lipschitz continuous with respect to $t \in[0, T]$,

$$
\rho(t, \mathbf{x})=\left\{\begin{array}{ll}
\rho_{s}\left(\mathbf{A}^{-1}(t, \mathbf{x})\right), & \mathbf{x} \in S(t) ; \\
\rho_{f}, & \mathbf{x} \in F(t),
\end{array} \text { for a.e. } t \in(0, T),\right.
$$

$\mathbf{u} \in C_{\text {weak }}\left(0, T ; V^{0,2}(\Omega)\right)$ and the following energy inequality holds

$$
\begin{gathered}
\frac{1}{2} \int_{\Omega} \rho|\mathbf{u}|^{2}(r) d \mathbf{x}+\int_{0}^{r}\left\{\int_{F(t)} 2 \mu_{f}|\mathbb{D} \mathbf{u}|^{2} d \mathbf{x}+\int_{\partial S(t)} \gamma\left|\mathbf{u}_{f}-\mathbf{u}_{s}\right|^{2} d \mathbf{x}\right\} d t \\
\leqslant \frac{1}{2} \int_{\Omega} \rho_{0}\left|\mathbf{u}_{0}\right|^{2} d \mathbf{x}+\int_{0}^{r}<\mathbf{g}, \mathbf{u}>d t \quad \text { for a.e. } r \in(0, T) .
\end{gathered}
$$

Let us point out that in D Gérard Varet ${ }^{17}$ it has been also studied the mixed case: Navier type condition on the boundary of the ball and Dirichlet condition on the boundary of the wall (the boundary conditions (5), (6)). The boundaries of the ball and the wall have been belonged to the class $C^{\infty}$. In this case it has been shown that the ball never touches the boundary of the wall for these boundary conditions. Nevertheless of the result, ${ }^{17}$ the contacts of the body and the boundary of the domain are available in Theorem 2.2, due to the low regularity of the boundaries $\partial \Omega \in C^{0,1}, \partial S_{0} \in C^{2}$. Moreover Theorem 2.2 is valid for any external force $\mathbf{g} \in L^{2}\left(0, T ;(K B(S(t))){ }^{*}\right)$. Let us refer to the example constructed by Starovoitov. ${ }^{11}$ In order to create a collision of the body with the boundary of the domain (in the case of non-slip conditions on the boundaries $\partial \Omega$ and $\partial S_{0}$ ), an appropriate external force from $H^{-1}$-space has been chosen. Regarding to the uniqueness of solutions we have to investigate two different situations: firstly we consider that the body $S(t)$ does not touch the boundary $\partial \Omega$ and secondly we admit the body contacts the boundary.

The unique solvability of the weak solution for the 2-D case was proved in the work of Glass $\mathrm{O}$ et al. ${ }^{16}$ The uniqueness result was obtained on the time interval as long as no collision occurs. It is wellknown that the uniqueness of the weak solution is not yet shown for the three-dimensional Navier-Stokes equations, then we can not expect to derive the uniqueness for 3D weak solution of system (1)(6). Concerning to the $3 \mathrm{D}$ uniqueness of strong solution, we remark that the local existence of unique strong solution and the weak-strong uniqueness have been obtained in the articles ${ }^{19}$ on the time interval where the strong solution exists.

In case that the body contacts the boundary, the situation is more delicate, since Definition 2.1 does not predict what may happen after the collision of the body $S(t)$ with the boundary $\partial \Omega$. In fact, equation (8) does not prescribe any rebound law. In the article $e^{20}$ two solutions have been constructed with different behaviors after collisions: the first describes the body hits the boundary $\partial \Omega$ and gets bounced back, and in the second solution the body and the boundary $\partial \Omega$ remain in contact after the collision. Therefore when the collisions happen the model must be more sophisticated.

It is not clear how to model correctly the situation at/after the collisions. It is still an open question. One natural way is to consider an elastic body and an elastic boundary instead of rigid ones. We can 
mention few results for the motion of an elastic body (and an elastic boundary) in a viscous fluid obtained by Boulakia $\mathrm{M},{ }^{21}$ Canic $\mathrm{S}^{22}$ and Kukavica I et al., ${ }^{23}$ where local-in-time existence results have been demonstrated as long as no collisions occur. The investigations of contacts of elastic bodies are not known to us.

\section{Sketch of the proof of theorem}

First we introduce an approximate scheme to system (1)-(6), using the idea that the "body+fluid" can be approximated by a nonhomogeneous fluid, having different values of viscosity in three zones: approximation of "body", approximation of Navier boundary condition (5) and "fluid" zone.

To construct such approximation problem we fix the following notations. For an open connected set $S \subset \mathbb{R}^{N}$, we define $\operatorname{dist}[\mathbf{x}, S]=\inf _{\mathbf{y} \in S}|\mathbf{x}-\mathbf{y}|, \quad d_{S}(\mathbf{x})=\operatorname{dist}\left[\mathbf{x}, \mathbb{R}^{N} \backslash S\right]-\operatorname{dist}[\mathbf{x}, S]$ for any $\mathbf{x} \in \mathbb{R}^{N},[S]_{\delta}=d_{S}^{-1}((\delta,+\infty))$ - the $\delta$-kernel of $S$ and ]$S\left[_{\delta}=d_{S}^{-1}((-\delta,+\infty))\right.$ - the $\delta$ - neighborhood of $S$.

As $\Omega$ is a bounded domain, we assume that $\Omega \subset]-L, L{ }^{N}=\mathcal{T}$ for a certain $L>0$. Let us extend the functions $\rho_{0}, \mathbf{u}_{0}$ and $\mathbf{g}$ by zero values on $\mathcal{T}$. Let us consider the characteristic functions $\xi(\mathbf{x})$, $\varphi_{0}(\mathbf{x})$ and $\chi_{0}^{\delta}(\mathbf{x})$ of the sets $\mathcal{T} \backslash[\Omega]_{2 \tau}, \quad S_{0}$ and $] S_{0}\left[\delta_{\delta} \backslash S_{0}\right.$, defined on the whole $\mathcal{T}$. Also we define $\rho_{0}^{\varepsilon \delta}=\left(1-\chi_{0}^{\delta}\right) \rho_{0}+\varepsilon \chi_{0}^{\delta}$.

Let $\sigma \in C^{\infty}(\mathbb{R})$ be a positive even function with pupportin $(-1,1)$, such that $\int_{\mathbb{R}^{N}} \sigma(|\mathbf{x}|) d \mathbf{x}=1$. We denote $\sigma^{\tau}(\mathbf{x})=\frac{}{N} \sigma\left(\frac{\mathbf{x} \mid}{)}\right)$ for any $\tau>0$. Now we can define the standard mollification of $\boldsymbol{u}$ on $\tau$ by

$\overline{\mathbf{u}}^{\tau}(t, \mathbf{x})=\int_{\mathcal{T}} \mathbf{u}(t, \mathbf{y}) \sigma^{\tau}(\mathbf{x}-\mathbf{y}) d \mathbf{y}, \quad \forall \mathbf{x} \in \mathbb{R}^{N}$, where extended by zero outside of a domain $\Omega$.

The approximation problem to system (1)-(6) consists of the transport equations

$$
\begin{aligned}
& \left.\partial_{t} \rho+\left(\mathbf{u}^{-\tau} \cdot \nabla\right) \rho=0, \quad \partial_{t} \varphi+\left(\stackrel{\mathbf{u}}{\tau}^{-\tau} \cdot \nabla\right) \varphi=0, \quad \partial_{t} \chi+\left(\stackrel{\mathbf{u}}{ }^{-\tau} \cdot \nabla\right) \chi=0 \quad \text { in }(0, T) \times\right] \mathcal{T}\left[_{\tau},\right. \\
& \left.\rho(0)=\rho_{0}^{\varepsilon \delta}, \quad \varphi(0)=\varphi_{0}, \quad \chi(0)=\chi_{0}^{\delta} \quad \text { in }\right] \mathcal{T}\left[{ }_{\tau},\right.
\end{aligned}
$$

and the momentum equation

$$
\begin{aligned}
& \int_{0}^{T}\left\{\int_{\mathcal{T}}\left[\rho \mathbf{u} \partial_{t} \mathrm{y}+\rho \mathbf{u}\left(\mathbf{u}^{-\tau} \cdot \nabla\right) \mathrm{y}-\xi_{\varepsilon} \mathbf{u y}-\mu_{\delta} \mathbb{D} \mathbf{u}: \mathbb{D} \mathbf{y}+\rho \mathbf{g y}\right] d \mathbf{x}\right. \\
& -\int_{\mathcal{T}}\left[{ }_{\tau} \zeta_{\varepsilon} \mathbb{D} \mathbf{u}^{-\tau}: \mathbb{D y}^{-\tau} d \mathbf{x}\right\} d t=-\int_{\mathcal{T}} \rho_{0}^{\varepsilon \delta} \mathbf{u}_{0} \mathrm{y}(0, \cdot) d \mathbf{x},
\end{aligned}
$$

which is valid for any test function $\mathrm{y} \in L^{2(N-1)}\left(0, T ; V^{1,2}(\mathcal{T})\right) \cap H^{1}((0, T) \times \mathcal{T}): \mathrm{y}(T, \cdot)=0$. Here

$\xi_{\varepsilon}=\frac{1}{\varepsilon} \xi, \quad \mu_{\delta}=\varphi+2 \mu_{f} \theta+\gamma_{0} \chi \int_{]} \mathcal{T}\left[_{\tau} \chi d \mathbf{x}, \quad \zeta_{\varepsilon}=\frac{1}{\varepsilon} \varphi, \quad \theta=1-(\varphi+\chi)\right.$ with the constants $\gamma_{0}=\frac{\gamma}{\left|\partial S_{0}\right|},\left|\partial S_{0}\right|=\int_{\partial S_{0}} 1 d \mathbf{x}$.

In relation (13) the "viscosity" term $\zeta_{\varepsilon}$ is an analog of penalization, introduced in $\mathrm{KH}$ offmann ${ }^{1}$ where the rigid body is replaced by a fluid, having high viscosity value. The first and second terms in the "viscosity" $\mu_{\delta}$ correspond to the "rigid" region and the fluid, respectively. The third term in $\mu_{\delta}$ defines a mixture region between the "body" and the fluid, which approximates the jump boundary term on $\partial S(t)$ in (8). The penalization $\xi_{\varepsilon}$ was developed in E Feireisl $1^{3}$ which is used here just for technical purposes. The solvability of this approximation problem (12)-(13) can be shown by Galerkin's method and theoretical results for transport equations. ${ }^{24-26}$

Next in the approximation problem we have to pass on limits with respect of the parameters $\varepsilon, \delta$ and $\tau$. These limits are based on the results for the transport equations. ${ }^{25}$

The first limit on $\varepsilon \rightarrow 0$ is related with a so-called "solidification" procedure in the zone of the non-homogeneous fluid, corresponding to the "body". This limit can be treated as in KH offmann', JA San Martin ${ }^{13}$ In the limit we obtain the motion of the rigid body in a viscous fluid, which occupies the domain $[\Omega]_{2 \tau}$.

In the second limit on $\delta \rightarrow 0$, we obtain the motion of the body already with a prescribed Navier boundary condition. Firstly we need to construct an appropriate set of test functions, depending on $\delta$. Then, using embedding results in cusp domains, we show that the third term of $\mu_{\delta}$ converges to the jump boundary term on $\partial S(t)$ in (8). The embedding results allow also to apply a compactness result in the convective term of (13) by using the approach of Proposition 6.1 in D Bucur ${ }^{27}$ (see also Lemmas 3.3, 4.9 and 4.10 in the article). ${ }^{28}$

Finallywe take the limit on $\tau \rightarrow 0$ being the regularization of the velocity $\mathbf{u}$. Using the techniques developed in the previous limit on $\delta \rightarrow 0$ we demonstrate our main result: Theorem 2.2.

The demonstration of Theorem 2.2 is a quite lengthy and technical one. The details can be found in NV Chemetov. ${ }^{29}$

\section{Acknowledgments}

The work of S Necasova was supported by Grant No. 16-03230S of GACR in the framework of RVO 67985840.

\section{Conflicts of interest}

Author declares that there is no conflicts of interest.

\section{References}

1. KH offmann, VN Starovoitov. On a motion of a solid body in a viscous fluid. Two dimensional case. Adv Math Sci Appl. 1999;9:633-648.

2. C Conca, J San Martin, M Tucsnak. Existence of solutions for the equations modelling the motion of a rigid body in a viscous fluid. Commun Partial Differential Equation. 2000;25:1019-1042.

3. E Feireisl, M Hillairet, Š Nečasová. On the motion of several rigid bodies in an incompressible non-Newtonian fluid. Nonlinearity. 2008;21(6):1349-1366

4. C Bost, GH Cottet, E Maitre. Convergence Analysis of a Penalization Method for the Three-Dimensional Motion of a Rigid Body in an Incompressible Viscous Fluid. SIAM J Numer Anal. 2010;48(4):1313-1337.

5. B Desjardins, MJ Esteban. Existence of weak solutions for the motion of rigid bodies in a viscous fluid. Arch in Rat Mech Anal. 1999;146(1):59-71.

6. MD Gunzburger, HC Lee, GA Seregin. Global Existence of Weak Solutions for Viscous Incompressible Flows around a Moving Rigid Body in Three Dimensions. J Math fluid mech. 2000;2(3):219-266.

7. T Takahashi. Analysis of strong solutions for the equations modelling the motion of a rigid-fluid system in a bounded domain. Advances in Differential Equations. 2003;8(12):1499-1532.

8. NV Judakov. The solvability of the problem of the motion of a rigid body in a viscous incompressible fluid. Dinamika Splošn Sredy. $1974 ; 18: 249-253$. 
9. TI Hesla. Collision of smooth bodies in a viscous fluid: A mathematical investigation. PhD Thesis, University of Minnesota, USA; 2005:159.

10. $\mathrm{M}$ Hillairet. Lack of collision between solid bodies in a $2 \mathrm{D}$ incompressible viscous flow. Comm Partial Differential Equations. 2007;32(7-9):1345-1371.

11. VN Starovoitov. Behavior of a rigid body in an incompressible viscous fluid near boundary. International Series of Numerical Mathematics. 2003; 147:313-327.

12. D Gérard Varet, M Hillairet. Regularity issues in the problem of fluid structure interaction. Arch Ration Mech Anal. 2010;195(2):375-407.

13. JA San Martin, VN Starovoitov, M Tucsnak. Global weak solutions for the two dimensional motion of several rigid bodies in an incompressible viscous fluid. Arch Rational Mech Anal. 2002;161(2):113-147.

14. J Neustupa, P Penel. Existence of a weak solution to the Navier-Stokes equation with Navier boundary condition around striking bodies. Comptes Rendus Mathematique. 2009;347(11-12):685-690.

15. G Planas, F Sueur. On the "viscous incompressible fluid + rigid body" system with Navier conditions. Annales de l'Institut Henri Poincare (C) Non Linear Analysis. 2014;31(1):55-80.

16. D Gérard Varet, M Hillairet. Existence of weak solutions up to collision for viscous fluid-solid systems with slip. Comm Pure Appl Math. 2014;67(12):2022-2076.

17. D Gérard Varet, M Hillaire, C Wang. The influence of boundary conditions on the contact problem in a 3D Navier Stokes flow. J Math Pures Appl. 2015;103(1):1-38.

18. O Glass, F Sueur. Uniqueness results for weak solutions of two-dimensional fluid-solid systems. Arch Ration Mech Anal. 2015;218(2):907-944.

19. HA Baba, NV Chemetov, Š Necasová. Strong solutions in $\mathrm{L}^{2}$ framework for fluid-rigid body interaction problem-mixed case. Analysis of PDEs. 2017:1-14
20. VN Starovoitov. Nonuniqueness of a solution to the problem on motion of a rigid body in a viscous incompressible fluid. J Math Sci. 2005; 130(4):4893-4898.

21. M Boulakia. Existence of weak solutions for the three-dimensional motion of an elastic structure in an incompressible fluid. J Math Fluid Mechanics.2007;9(2):262-294.

22. S Čanic, B Muha. Existence of a weak solution to a nonlinear fluidstructure interaction problem modeling the flow of an incompressible, viscous fluid in a cylinder with deformable walls. Arch Ration Mech Anal. 2013;207(4):919-968.

23. I Kukavica, A Tuffaha, M Ziane. Strong solutions to a nonlinear fluids-structure interaction system. $J$ of Differential Equations. 2009;247:1452-1478.

24. SN Antontsev, AV Kazhikhov, VN Monakhov. Boundary Value Problems in Mechanics of Non-homogeneous Fluids. In: Studies in Mathematics and its Applications. 1st Edn. Amsterdam, Netherlands: North-Holland Publishing Co; 1990:309.

25. F Boyer. Trace theorems and spatial continuity properties for the solutions of the transport equations. Differential Integral Equations. 2005;18(8):891-934.

26. R Temam. Navier-Stokes equations. Theory and numerical analysis. Providence, Rhode Island: AMS Chelsea publishing; 1984. 408 p.

27. D Bucur, E Feireisl, Š Nečasová, et al. On the asymptotic limit of the Navier-Stokes system with rough boundaries. J Differential Equations. 2008;244(11):2890-2908.

28. NV Chemetov, ک̌ Nečasová. The motion of the rigid body in viscous fluid including collisions. Global solvability result. Nonlinear Analysis: Real World Applications. 2017;34:416-445.

29. NV Chemetov, Š Nečasová, B Muha. Weak-strong uniqueness for fluidrigid body interaction problem with slip boundary condition. Analysis of PDEs. 2017. p. 1-18. 